\title{
PERSONALIZATION ALGORITHMS - LIMITING THE SCOPE OF DISCOVERY? HOW ALGORITHMS FORCE OUT SERENDIPITY
}

\author{
Mihail Vuzharov \\ New Bulgarian University, Bulgaria \\ vuzharov@gmail.com
}

\begin{abstract}
The Digital has become ubiquitous and inevitable. Each day, fewer non-digitals remain, as others become digital immigrants, and finally being succeeded by digital natives. Billions of devices are now connected, as remote access and IoT-added-value have become commonplace. Cloud services have supplanted old-school digital products, personal data has become more valuable than most other resources, while our attention span has been shrinking, constantly besieged by millions of signals.

It is now virtually impossible for anyone to exist outside of the Digital; it is virtually impossible not to rely on online services, not to have our data collected, not to have information tailored especially for our personal consumption, based on our unique digital footprints. UX Design paradigms have been shifting, moving us further from simple interaction, departing from on-screen interfaces, and simultaneously eliminating the need for a
\end{abstract}

Digital Age in Semiotics \& Communication, Vol. I, No. 1, Spring 2018, Pp. 19-33 
user's encyclopedic competence (as per Eco) and even going past navigational competence (as per Bankov).

Communication structures define communication outcomes. Communication structures literally shape our world, as Benedict Anderson would argue. While his analysis turns to the printing press as a causal mechanism for the formation of the nation states, one could argue that the algorithm-based structure of information delivery means a departure from the potential for serendipitous discovery, changing our systems of expectations, the way we think, and the way we perceive the world.

If the entire system is based on our past, a mirror image of ourselves, this would mean that we are more likely to receive answers pertaining to a world that is entirely within our scope. The farther we depart from encyclopedic competence, and then from navigational competence (where we were at least able to browse into areas unknown), the farther we are moving from the unfamiliar. There is an event horizon, the information beyond which is completely outside our reach, and this event horizon is more and more tightly enclosing us.

Essentially, our entire information inflow is based on a user model, derived by various algorithms, deep learning mechanisms and AI systems - a veritable black box, which, in turn, weaves a personalized and unique Dynamic Text for a very special Echian "model reader" - the "model user". We will try to demonstrate how this relationship may lead to a limited outlook.

Keywords: Social media, algorithms, suggestion, discovery, encyclopedia

While Internet users may agree that their online experiences vary, as they generally tend to, the truth is somewhat more complex - and far less transparent. The Internet is different for each of us; in fact, since between 2006 and 2010, the Internet has become almost fully personalized. This paper looks at the structure of today's Internet - more precisely, User Experience Design, personalization and recommendation, algorithms and artificial intelligence - and at the way its information delivery design influences users. Far from claiming the discovery of a novel phenomenon, our text will attempt to apply semiotic methods to existing hypotheses and analyses, in order to help clarify how certain subtle (at first glance) changes in structure and infrastructure may have led to rather profound changes in individual perception, and thus in the very fabric of society.

Mundane Internet use can be described as serving a few main purposes: connecting with others (including email, chat, social media); getting infor- 
mation (including news, research, finances); shopping (for products and services); and entertainment (video, music, gaming). (Meyen 2010). These activities tend to take place on a surprisingly limited number of platforms, many of them owned by Google (including local versions of the search engine, YouTube, Gmail and other Google services) ${ }^{1}$ and Facebook (including Messenger, Instagram, Whatsapp) ${ }^{2}$.

Therefore our analysis will concentrate on prominent players, mostly Google and Facebook, seeing as they command (at least as of today) the largest piece of the Internet pie, also due to the fact that they either tend to set the trends, or purchase other trend-setters in the market and absorb or adapt their models. We may also mention some other large players such as Apple and Amazon, or trending developments, such as IoT, autonomous cars, etc., but only as much as they fit within the scope of our current analysis.

\section{Personalization}

Personalization is typically presented in terms of relevance and user satisfaction, i.e. showing only search results (in Google's case), social media posts (Facebook), product recommendations (Amazon), etc., which are relevant (i.e. useful, appropriate) to the specific user. This is a leading feature of most online services, since relevance is one of the most important keywords in what we now call the Attention Economy.

This need for relevance is born out of the sheer amount of data available online; the Internet has come to contain a veritable - and unfathomable universe of information, feeding the need for contextual reduction of available information down to applicable micro-universes and, effectively, their rendition into legible discourse universes (such as, for example, the list of search results for a certain search term by a specific Google user).

In order to fulfill their stated purpose (a topic we will revisit later) and to deliver a better user experience, online services, such as Facebook and Google, utilize proprietary algorithms, meant to provide an improved and customized information flow to their users.

Google launched its personalized search in 2005, first in beta, then only to subscribed and logged-in users. This new search would take into account all of the information available about the logged-in user and would attempt to provide the most relevant search results to any search query. Then, in

\footnotetext{
${ }^{1}$ Similarweb - 100 Top Websites (last accessed September 2017 from https://www.similarweb.com/top-websites)

${ }^{2}$ Comscore Mobile Metrix (last accessed September 2017 from https://www.comscore. com/Products/Audience-Analytics/Mobile-Metrix)
} 
2009, Google launched the personalized search to all users, logged-in or not. Respectively, this would mean that, while there is more personal information about logged-in users, the algorithm still has plenty of data available in order to narrow down the search results for anyone - data such as date and time, location, browser, device, pages visited, scrolling patterns, as well as any other data Google's cookies manage to collect. ${ }^{3}$ In fact, Google's Chrome is now the most widely used browser across almost all platforms, which allows its parent company to collect even more information about its users.

Similarly, Facebook introduced its NewsFeed in 2006 - the familiar stream of "stories" coming from friends and pages that every Facebook user knows so well. While initially a purely chronological mass of consequent items, Facebook changed the logic behind the Newsfeed in 2011, due to the ever-increasing number of "stories" avalanching the users - reportedly, over 2000 items per user per day; the NewsFeed was to become a highly personalized affair, delivering only those stories the algorithm deems worthy of the user's attention, based on her past interactions (with her Facebook friends, pages, topics, etc.) and on her similarity to other users. ${ }^{4}$

While these developments have indeed managed to improve the end-user's experience, they have also defined a certain structure, a pre-set mode of information retrieval and communication: both services have achieved their current level of personalization by creating a complex model of the user and serving data (be it status updates and links, or search query results) based on that model.

The way this user model is created generally relies on three (and-judging by patents filed by some of the companies-soon to be four) main paradigms: user behavior, collaborative filtering, inference/extrapolation (and soon - disposition/emotion analysis).

Behavior analytics is a historical, statistical analysis based on past actions and preferences by the specific user. It requires the capture and aggregation of large quantities of raw data (signals) across all of the user's connected applications and devices. While a logged-in user is easier to track across multiple devices, it is still possible to collect plenty of information even for users who are not logged into the platform. Marketing and analytics services, such as Acxiom, have devised methods that allow them

\footnotetext{
${ }^{3}$ Personalized Search for Everyone. 2009. (last accessed September 2017 from https:// googleblog.blogspot.it/2009/12/personalized-search-for-everyone.html)

${ }^{4}$ The Evolution of Facebook News Feed. (last accessed January 2018 from http://mashable. com/2013/03/12/facebook-news-feed-evolution/\#d7dXN2ZeQPqf)
} 
to match figures from different user sessions and, subsequently, aggregate immense volumes of data. (Pariser 2011).

Collaborative filtering, on the other hand, relies on grouping users into clusters based on their similarities. It is based on overt social characteristics and processes, such as rating certain songs (on Spotify) or purchasing certain products (on Amazon). These user signals can then be interpreted as an approximation of the user's perception of the respective realm and her association (by similarity) to an abstract cluster of users who exhibit similar attitudes. Such filtering mechanisms largely power recommendation services, such as Spotify's Discover Weekly playlists; they look at a cluster of similar users and recommend highly rated items to those users who have not yet encountered them (or, at least, for whom there is no data regarding such encounters in their user profiles).

In contrast to collaborative filtering, inferential methods are based on covert social characteristics and processes, and often rely on psychographics and statistical data drawn from large populations. They are based on the analysis of hundreds of thousands of records in order to extrapolate additional details about users. For example, using explicit Facebook page likes in order to reach certain conclusions (e.g. if the user likes Nutella, she is classified as a sweet-tooth, chocolate lover). However, this method also relies on implicit traits in order to classify users (e.g. if the user is male and his Facebook page-likes include "Wicked the Musical", the "No H8" campaign, and other human rights campaigns, he may be classified as gay). ${ }^{5}$

Finally, we should mention the newest trend in user profiling, namely dispositional analysis. While this technology is still being tested and finetuned, it does show great promise (for marketing purposes). What this method aims to achieve is to recognize the user's current mood, in order to surface adequate information (or to render an applicable discourse universe). It is yet another layer of the user model, which would allow an app or service to use the built-in webcam (or other available instrument) of a user's device, analyze her face (or why not pulse, bodily temperature, etc.), and eventually display information, which would harmonize with the user's emotional state (or would, in more unethical circumstances, take advantage of her momentary emotional state).

Essentially, the entire premise of personalization is an approach built upon the user's observed behavior, her user model constantly being updat-

\footnotetext{
${ }^{5}$ Halliday, Josh. 2013. Facebook users unwittingly revealing intimate secrets, study finds (last accessed January 2018 from https://www.theguardian.com/technology/2013/mar/11/ facebook-users-reveal-intimate-secrets)
} 
ed with regard to the persistent flow of new behavioral data. In other words, the user model is an attempt at a digitally reconstructed approximation of the user's individual encyclopedic competence and system of expectations.

\section{Algorithms and Data}

Collecting all of the abovementioned data is a gargantuan task. So much so that collecting, analyzing, and utilizing this data has become one of the most lucrative fields. Little wonder this phenomenon is dubbed Big Data. Actually, data has been called the most valuable resource, effectively displacing even oil. ${ }^{6}$

In fact, although Google is generally regarded as a search company while Facebook is thought of as a social network, they both depend almost entirely on data, all of it collected from the Internet users who peruse their free services. What they do with this data is straightforward: they sell it. This statement may sound rather blunt, so we will peek into the process.

Both companies' revenues are generated mostly by advertising. In fact, this is how - and especially why - they offer such high-quality services for free: their model, albeit not sound from a classical free-market point of view, depends on subsidizing the free side (social, search, chat, entertainment, office suite, etc.) of their business via revenues from the paid side (advertising), while, on the other hand, the free side accumulates the data needed for the smooth (and profitable) functioning of the paid side. (Srnicek 2017).

This internal structure, therefore, requires that the services these companies provide for free are of high quality. Even more importantly, they need to make sure that the users are not only satisfied, but drawn to the services, addicted if possible, and even dependent on them. In some cases, the companies practice customer lock-in - making it prohibitively expensive (not necessarily in financial terms) for a user to switch to a different service. Far from malevolence, this actually demonstrates strong business acumen. Which brings us back to the topic of relevance: a well-functioning, lightning-fast and relevant web service - one able to avoid the notorious attention crash - is a good service.

Google managed to replace Yahoo and AltaVista as the most used search engine by replacing their tree-based, dictionary-style search process with a rhizome-based (as Eco would regard it), encyclopedia-style search algorithm, while Facebook enjoys the benefits of the network effect to the

\footnotetext{
${ }^{6}$ The World's Most Valuable Resource is No Longer Oil but Data. 2017. (last accessed January 2017 from https://www.economist.com/news/leaders/21721656-data-economy-demands-new-approach-antitrust-rules-worlds-most-valuable-resource)
} 
extent that not using Facebook services bears a relatively high social price. With the added value of high quality personalization across the board, the two services have achieved supremacy over most other end-user services, while their unsurpassed ability to collect, analyze, package and sell user data has made them into powerful one-stop-shops for advertisers.

While this development bodes well for the companies, it has a frustrating side effect: it turns the algorithms, which work behind the scenes to provide the personalized information flow to users, into double agents. Their loyalty has no alternative but to shift from the (freeloading) user to the (paying) client, at least to some extent, seeing as these high-quality free online services are, after all, only a part of actual commercial entities (and quite gainful ones, at that), rather than not-for-profit organizations (as a counterpoint, we should mention Wikipedia, which has managed to remain free and independent from advertisers and the corresponding market forces). Since the objective of these companies (both companies are listed) is first and foremost profit, business interests come first.

An additional deficiency of algorithms tends to remain unnoticed, although recently there has been an upsurge in research on the topic: algorithms are not neutral, are imperfect, and are subject to their creators' fallibilities. (O'Neil 2017). This can be related to the point above: when an algorithm is created to serve a certain primary purpose (while also satisfying a certain other secondary requirement), it will tend to lead to skewed outcomes, as opposed to a neutral algorithm (which is, most likely, unconceivable, since this would require a programmer without competing allegiances).

What this implies is that, if the ideal (neutral) algorithms were applied to the semantic micro-universe of a specific user's web experience, in order to reduce the available information down to an applicable discourse universe, they would produce a snapshot that would correspond only to the user's properties and desires. However, since algorithms are imperfect, they would tend to render a more limited version of this potential discourse universe, since it would have to meet the conditions for two separate agents - the user and the algorithm's distortion (due to its primary purpose, its creator's fallibilities, etc.).

\section{Structure and Control}

What we have depicted in the previous section is, essentially, a form of control. However, it must be re-stated that this is not a malicious design; rather, what we are observing is a healthy (from an economical point of 
view) pursuit of optimization and market domination, which, however, cannot be realized without the shaping of the information delivery mechanisms - i.e. the design of the algorithms. On the one hand, the algorithms must be designed in a way that will increase the (perceived) value for the users, in order to attract them to and retain them within the service. And on the other hand they concentrate on those traits which can be most useful to the highest bidder for the advertising space offered on the services' real estate.

Additionally, but not independently from the previous points, the services do shape the rules for their users. A simple illustration would be Facebook's "Like" button - one of the UX (user experience) affordances that Facebook's algorithms rely on, when modeling a user's NewsFeed and her entire potential semantic universe. This button is utilized when a user wants to interact positively with a certain piece of information on Facebook, be it a status update, photo of a cat, a shared link, etc. This creates an instant semiotic contradiction - "things she likes" vs. "things she doesn't like" (reGreimas Semiotic Square spectively $\mathbf{S}_{1}$ and $\sim \mathbf{S}_{1}$ in Fig. 1$)^{7}$. However,

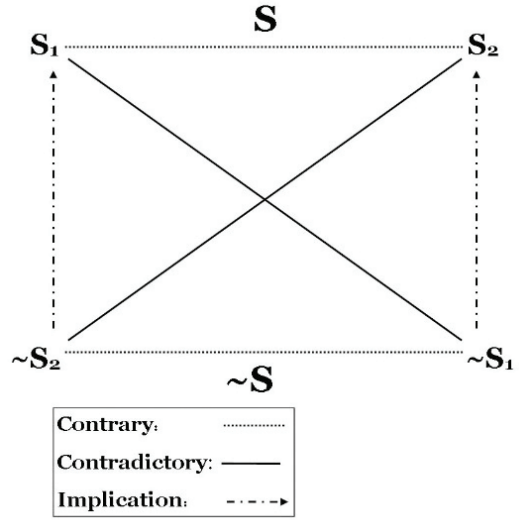

Figure 1: Semiotic Square even with the recent introduction of Facebook "reactions" (the new ones being "Love", "Haha", "Wow", "Sad", "Angry") one will be unable to complete the semiotic square with the obviously missing "things she dislikes" $\left(\mathbf{S}_{2}\right)$ and "things she doesn't dislike" $\left(\sim \mathbf{S}_{2}\right)$. Indeed, it would be hard to have the latter without the former, however "dislikes" are impossible to monetize in the existing ad-based profit model. Essentially, what we have here is an incomplete structure serving as the user model's foundation, which is then used as the filter through which the user perceives the universe.

Additionally, Facebook and Google both prioritize items with which the user seems more likely to interact, based on her user model. As stated earlier, this is a matter of relevance. This approach, however, has been criticized profusely. One criticism in particular uses a facetious illustration: if roads functioned the way Facebook does, and if we were to take into account the

\footnotetext{
${ }^{7}$ EmmaSofia515-Own work, Public Domain, https://commons.wikimedia.org/w/index. php? curid $=10232752$
} 
way people react to accidents (rubbernecking), then roads would have to provide us with more car crashes. Essentially, this model tends to conflate popularity with legitimacy.

The substantive design of this infrastructure results in a lopsided representation of reality. As has been stated time and again, the medium shapes our perceptions. Communication structure defines communication outcomes; as Benedict Anderson would argue, it literally shapes our world. Just as he analyzed the influence of the printing press and capitalist printing on the formation of nation states, here we are looking at the algorithm- and recommendation-based structure of information delivery and the possibly resulting changes in our systems of expectations, in the way we think, and the way we perceive the world. "The medium is the message."

\section{User Experience Design and the Text}

As we alluded to earlier, the abovementioned algorithm design and the fight for relevance are all facets of User Experience (UX) design. While the notion of UX has only recently become familiar to the public (although it was introduced by Donald Norman of the "Nielsen Norman Group" in the 90s), the field has actually been around for a long time, variants of it appearing under different names - including Interaction Design, User Interface Design, Usability, Human-Computer Interaction (HCI), and even Clarisse de Souza's Semiotic Engineering. (de Souza 2004). UX has been evolving parallel to technology, as its main stated purpose is to improve the experience of the user's interaction with products or services - so its roots can be traced back to the industrial age.

What we have been experiencing recently is the migration of UX away from the screen (or monitor) and closer to direct experience - or, as de Souza would put it, a decrease in articulatory and semantic distance. (de Souza 2004, 100). The goal is, essentially, to make it easy, intuitive and hassle-free for users to achieve their goals through the design language of the user interface of a product or service.

Until recently, during the Internet age's initial stages (when search engines were still modelled after the Porphyrian tree), navigating through the vast and constantly growing universe of information depended on the user's (Echian) personal encyclopedic competence: she had to understand what to look for and use her memory (recall Eco's letter to his grandson "Learn to memorize") ${ }^{8}$. Eventually, with advances in UX design, retrieving

\footnotetext{
${ }^{8}$ Eco. Umberto. 2014. Caro nipote, studia a memoria. (last accessed January 2017, from http://espresso.repubblica.it/visioni/2014/01/03/news/umberto-eco-caro-nipote-studiaa-memoria-1.147715?refresh_ce)
} 
information from the Internet became rather a matter of navigational competence: the user only had to know where to look. (Bankov 2010). Now, with advances in AI decreasing the distance between request and result, such as with Google Now, Apple's Siri, Amazon's Alexa, Microsoft's Cortana, etc. services, which are, arguably, still in their infancy - users need to rely on what could only be called an inquisitorial-processual competence: the user must only figure out how to frame the query in order to achieve the desired result. However, as these services mature, as Natural Language Processing moves forward, and as the Internet of Things (IoT) and more autonomous technologies such as self-driving vehicles are developed, we may move to a post-competence stage, where the interface has become invisible (and indistinguishable) and the articulatory and semantic distance has virtually been obliterated.

As can easily be observed, the predominant model of Internet use has changed immensely: from a single version for all users, through several steps of increasing personalization, to a fully personal Internet tailored for every user based on his or her user model, and delivered by a connected interactive agent with a minimalistic user interface, such as Amazon's Alexa. Essentially, our personal Internet is a complex Dynamic Text, dynamically woven for us (through with nary a sign of awareness on our side) - and only meant for our eyes (or ears) - and exclusively based on our very selves, on an immense set of parameters, opaquely aggregated by an ever-developing and constantly progressing multitude of algorithms, in order to re-create our sets of expectations: our user model.

\section{A Semiotic Model}

Our personal Internet's Dynamic Text, then, would be authored by way of the user model and intended to be read by the ideal actuation of the user model, namely the model user (recall Eco's Model Reader).

In order to arrange the process more legibly, let us turn to Eco's three intentions, as analyzed by Valentina Pisanti: the intentio auctoris (what the empirical author intends to say), the intentio operis (what the text wants to say with reference to its underlying signification system and by virtue of its textual coherence) and the intentio lectoris (what readers make the text say with reference to their own system of expectations, their wishes, drives, beliefs, and so on). (Pisanty 2015, 54). Additionally, to clarify the essence of the intentio operis, Pisanti maps the three intentions onto Perice's triadic model: 
[We] could try the experiment of projecting the three intentions onto the vertices of Perice's triangle. The intentio auctoris would correspond to the Dynamic Object ("really efficient but not effectively present," and therefore knowable only through the signs that represent it), the intentio lectoris to the Interpretant (the effect the text has on the interpreter's mind and ensuing behavior), the intentio operis with the Immediate Object ("the Object as represented in the sign," i.e., the communicative intention as it is represented by the text), while the expressive manifestation of the text would coincide with Peirce's Representamen.

The intentio auctoris and the intentio lectoris are psychological events, unknowable in themselves unless they are displayed as signs, and yet quite easy to conceive of as 'the cognitive activity that goes on in the author's and in the reader's minds.' The intentio operis acts as an interface between the two: on the one hand it is determined by the intentio auctoris (which, like the Dynamic Object, is the first mover of semiosis) and carries traces of it, and on the other it kindles an indefinite number of effects in empirical readers.

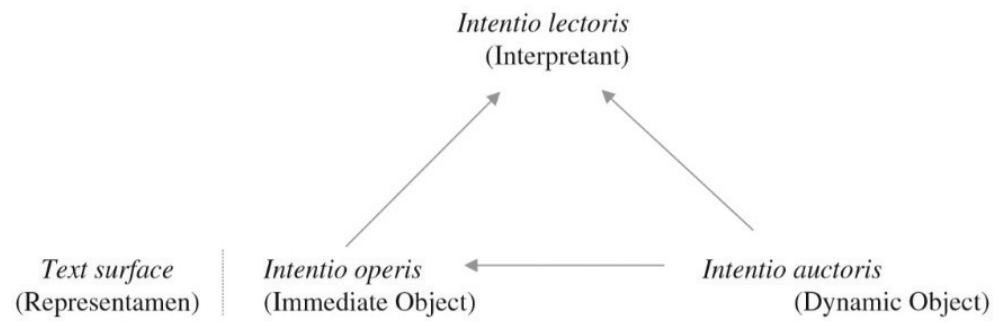

Figure 2: The above image represents a "normal" text, with a "normal" author and a "normal" reader.

It is very difficult to define the precise semiotic nature of this linking element (as it is difficult to define Peirce's Immediate Object). On the author's side, the intentio operis is a necessarily selective representation of an indefinite continuum of mental events that are channeled within the author's intention to produce certain cognitive effects on somebody through a communicative strategy (the Model Author, in fact). On the reader's side, the intentio operis is the matrix that generates all the possible interpretations of the textual Representamen (the Model Reader, possibly?)(.) (Pisanty 2015, 55). 
Our primary interest is the triadic relationship of the three Echian intentions, represented in the diagram above, as opposed to the Peircean fundamental, which Pisanti has utilized as a stepping stone.

Based on our reflections earlier in this text, it can be suggested that there would exist a feedback loop, where the intentio auctoris would ideally directly feed into the intentio lectoris (since the reader's own model is the basis of the Dynamic Text - authoring it, in a way). However, as we have already argued, the user model is skewed: the intentio auctoris has been intercepted and adulterated by an external agent - the algorithm (with its inherent biases and imperfections) which acts as a sieve, effectively rendering the intentio auctoris a debasement of the model of the intentio lectoris (Fig. 2). Thus, the empirical reader is presented with a localization, a specific (probably unique) frame of the encyclopedia that is otherwise available, the frame having been fashioned to suit the model user created (and compromised) by the algorithms (due to the nature of information that is important to the Services that collect it and feed it into the model itself). In fact, the External Agent appears to have violated the relationship between the three intentions, as it has positioned itself in such a way as to influence each of them: as it collects data from an initial state of the intentio lectoris, it creates a contaminated version of the potential intentio auctoris, essentially rendering a Dynamic Text whose intentio operis reflects the distortions brought into the cycle. Effectively, the model user, based on a reductionist user model is but a facsimile of the empirical user.

In most day-to-day communication, "the interpreter's main objective is to identify the Intentio auctoris starting from the perceptible clues which are present in the form of Intentio operis". (Pisanty 2015, 58). However, in day-to-day use of the Internet, the interpreter is unaware that she is, in fact, being presented with a Dynamic Text, that there is an author (of sorts), and that she is only looking at a modified subset of the available information.

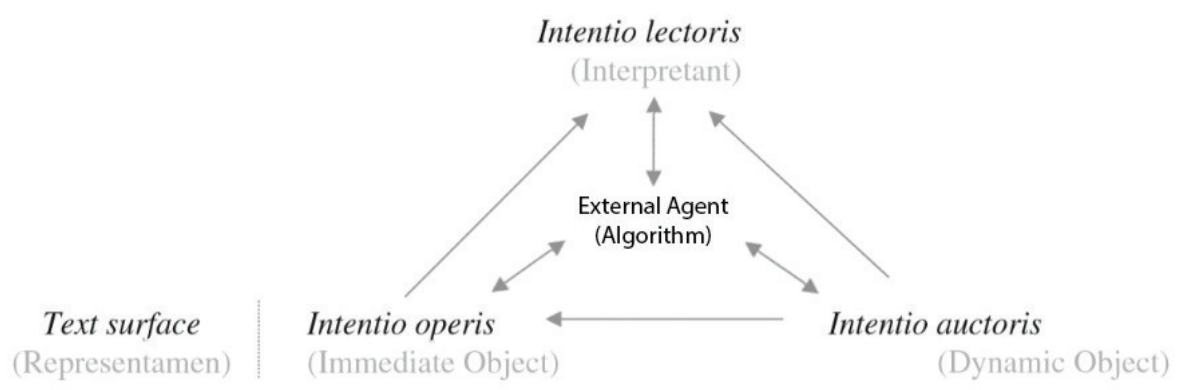

Figure 3: Illustrating the external agent (algorithm) and its effect on the model 
Naturally, one could argue that the better the algorithm, the better the model, and the more perfect the representation of the user. Essentially, the most perfect algorithm would be able to provide information which corresponds ideally to the user's own encyclopedic competence - no more, no less. Effectively, this is a self-perpetuating and self-limiting system. Such a structure would minimize serendipity, or chance discovery and productive error. The farther we depart from encyclopedic competence, and then from navigational competence (where we were at least able to browse into areas unknown), the farther we are moving from the unknown.

Only information from the known universe will be available to the user, while all else sits, unattainable, behind an event horizon which, counterintuitively and almost paradoxically, shrinks around the user as the algorithms become ever better at knowing her.

\section{A Short Afterword}

This text is, admittedly, looking at a greatly refined depiction of the way we consume media and the way our environment promotes the construction of our worldview. Such distillation is a prerequisite when trying to construct a valid model. Still, it would be sensible to note the fact that, while the self-perpetuating and self-limiting model we have described may indeed result in departure from serendipitous discovery, human beings, as a rule, do exist in societies and would hardly be as restricted in their sources of information as our analysis may illustrate.

Additionally, some of the companies mentioned in the text have been attempting to correct for certain aberrations their services have been shown to exhibit - for example, Google already allows users to review and edit some of the building blocks of their user profile at myactivity.google. com (although, as many UX designers know well, an option only exists as much as users are aware of it and willing to use it). Additionally, some services rely on human-curated content parallel to algorithm-created content - namely some Spotify playlists created by experts, or Yahoo! News articles which are manually selected to appear, due to their overall importance, as judged by actual human news editors.

It is our hope that grim forecasts, claiming humanity has surely headed towards a dystopian future fully controlled by a handful of extremely powerful supranational corporations, will prove false, as will visions of civilization depicted in fictitious discourses such as Netflix's "Black Mirror" series. However, we do believe that simply considering these issues is far from useless, as it can serve as our collective "things we dislike," in order for us to know what we should strive for instead. 


\section{References}

Anderson, Benedict. 2016. Imagined Communities: Reflections on the Origin and Spread of Nationalism. New York: Verso

Bankov, Kristian. 2010. Cultures of navigation versus cultures of erudition. Lexia Vol. I (September 2010), 103-123

Bankov, Kristian. 2017. Eco and the Google Search Innovations, in Thellefsen, T. and Sørensen, B. (eds) Umberto Eco in His Own Words, Berlin: De Gruyter Mouton, pp. 119-126.

Bergstein, Brian. 2017. We Need More Alternatives to Facebook. (last accessed January 2018 from https://www.technologyreview.com/s/604082/ we-need-more-alternatives-to-facebook)

Bogost, Ian. 2017. For Google, Everything is a Popularity Contest. (last accessed January 2018 from https:/www.theatlantic.com/technology/archive/2017/06/for-google-everything-is-a-popularity-contest/531762)

Eco, Umberto. 1978. A Theory of Semiotics. Bloomington: Indiana University Press

Eco, Umberto. 1986. Semiotics and the Philosophy of Language. Bloomington: Indiana University Press

Eco, Umberto. 2002. Serendipities: Language and Lunacy. London: Phoenix.

Eco, Umberto. 1991. The Limits of Interpretation. Bloomington: Indiana University Press

Eco, Umberto. 1979. The Role of the Reader. Bloomington: Indiana University Press

Knight, Will. 2017. The Dark Secret at the Heart of AI. (last accessed January 2018 from https://www.technologyreview.com/s/604087/thedark-secret-at-the-heart-of-ai/)

O'Neil, Cathy. 2016. The Dystopian Future of Price Discrimination. (last accessed January 2018 from https://www.bloomberg.com/view/articles/2017-03-16/the-dystopian-future-of-price-discrimination)

O’Neil, Cathy. 2017. Weapons of Math Destruction: How Big Data Increases Inequality and Threatens Democracy. New York: Broadway Books. Ebook. 
Orszag, Peter. 2017. People Lie, But Search Data Tell the Truth. (last accessed January 2018 from https://www.bloomberg.com/view/articles/2017-05-09/people-lie-but-search-data-tell-the-truth)

Pariser, Eli. The Filter Bubble: What the Internet is Hiding from You. London: The Penguin Press, 2011. Ebook.

Pisanty, Valentina. 2015. From the model reader to the limits of interpretation. Semiotica Vol. 206 (2015), 37-61

Sieckenius de Souza, Clarisse. 2004. The Semiotic Engineering of Human-Computer Interaction. Cambridge: The MIT Press.

Srnicek, Nick. 2017. Platform Capitalism. Cambridge: Polity Press.

Van Dijck, Jose. 2013. The Culture of Connectivity: A Critical History of Social Media. New York: Oxford University Press

Viner, Katharine. 2016. How technology disrupted the truth. (last accessed January 2018 from https://www.theguardian.com/media/2016/ jul/12/how-technology-disrupted-the-truth)

Wall Street Journal, The. 2016. Blue Feed, Red Feed: See Liberal and Conservative Facebook, Side by Side. (last accessed January 2018 from http://graphics.wsj.com/blue-feed-red-feed/) 